\title{
Magnetic Energy Release in Relativistic Plasma
}

\author{
Hiroyuki R. Takahashi ${ }^{1}$ and Ken Ohsuga ${ }^{2}$ \\ ${ }^{1}$ Center for Computational Astrophysics, National Astronomical Observatory of Japan, \\ Osawa, Mitaka, Tokyo 181-8588, Japan \\ email: takahshi@cfca.jp \\ ${ }^{2}$ National Astronomical Observatory of Japan, \\ Osawa, Mitaka, Tokyo 181-8588, Japan
}

\begin{abstract}
The efficiency of the energy conversion rate in the relativistic magnetic reconnection is investigated by means of Relativistic Resistive Magnetohydrodynamic (R2MHD) simulations. We confirmed that the simple Sweet-Parker type magnetic reconnection is a slow process for the energy conversion as theoretically predicted by Lyubarsky (2005). After the Sweet-Parker regime, we found a growth of the secondary tearing instability in the elongated current sheet. Then the energy conversion rate and the outflow velocity of reconnection jet increase rapidly. Such a rapid energy conversion would explain the time variations observed in many astrophysical flaring events.

To construct a more realistic model of relativistic reconnection, we extend our R2MHD code to R3MHD code by including the radiation effects (Relativistic Resistive Radiation Magnetohydrodynamics R3MHD). The radiation field is described by the 0th and 1st moments of the radiation intensity (Farris et al. 2008, Shibata et al. 2011). The code has already passed some one-dimensional and multi-dimensional numerical problems. We demonstrate the first results of magnetic reconnection in the radiation dominated current sheet.
\end{abstract}

Keywords. magnetohydrodynamics, radiation, relativity

\section{Introduction}

Magnetic reconnection is one of the most important subjects in the studies of space, laboratory, and astrophysical plasmas. Recently, it has been recognized that the magnetic reconnection plays an essential role for energy conversion in astronomical compact objects, such as neutron stars (Kennel \& Coroniti 1984), soft gamma-ray repeaters (Masada et al. 2010), and gamma-ray bursts (Drenkhahn 2002, McKinney \& Uzdensky 2010, Zhang \& Yan 2011). However, there are a few theoretical and numerical studies on the relativistic magnetic reconnection (Lyutikov \& Uzdensky 2003, Watanabe \& Yokoyama 2006, Zenitani et al. 2010, Zanotti \& Dumbser 2011).

We performed 2-dimensional Relativistic Resistive Magnetohydrodynamic (R2MHD) simulations to study the energy conversion in the magnetic reconnection. Then, we developed the Relativistic Resistive Radiation Magnetohydrodynamic (R3MHD) code to study the radiation effects. In this paper, we show the first results of the R3MHD simulation.

\section{Results}

We first perform R2MHD simulations of the magnetic reconnection with a uniform resistivity model. Then, Sweet-Parker type magnetic reconnection is realized in the relativistic plasma. In this case, most of the magnetic energy is converted into the thermal 


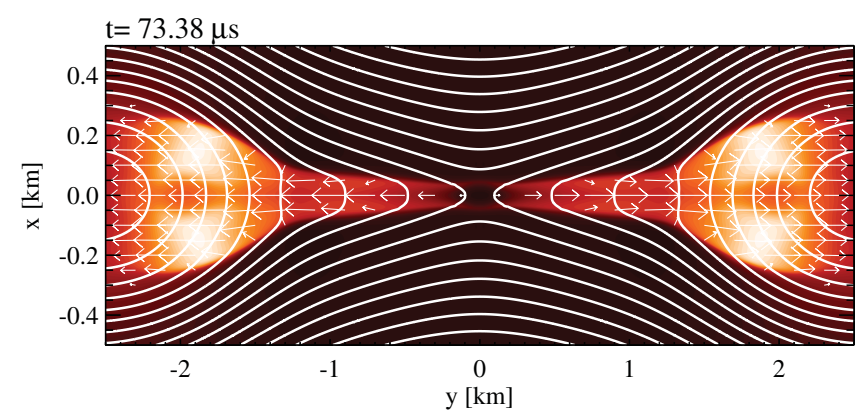

Figure 1. First numerical results of R3MHD simulation on the Petschek type magnetic reconnection. Color and arrows show the radiation energy density and flux in the observer frame, while the curves show the magnetic field lines.

energy by the Ohmic dissipation, resulting in the formation of a hot $\left(k_{B} T>m c^{2}\right)$ outflow. Since the excess of the thermal energy leads to increasing the plasma inertia, the outflow speed is only mildly relativistic (Lorentz factor $\sim 1$ ). The reconnection rate strongly depends on the magnetic Reynolds number $R_{M}$ as $\simeq R_{M}^{-0.5}$, as well as that in the non-relativistic magnetic reconnection (Komissarov 2005, Takahashi et al. 2011).

When the localized resistivity model is adopted, the Petschek type magnetic reconnection is realized in the relativistic plasma (Watanabe \& Yokoyama 2006, Zenitani et al. 2010, Zanotti \& Dumbser 2011). Figure 1 shows the first results of R3MHD simulation on the relativistic magnetic reconnection. We found that the radiation energy density is enhanced inside the reconnection outflow in our model.

\section{Acknowledgement}

Numerical computations were carried out on Cray XT4 at the Center for Computational Astrophysics, CfCA, at the National Astronomical Observatory of Japan, on Fujitsu FX-1 at the JAXA Supercomputer System (JSS) at the Japan Aerospace Exploration Agency (JAXA), and on T2K at the University of Tokyo. A part of this research has been funded by MEXT HPCI STRATEGIC PROGRAM and supported by Ministry of Education, Culture, Sports, Science, and Technology (MEXT) for Research Activity Start-up (HRT) 23840045, and for Young Scientist (KO) 20740115, 23340040.

\section{References}

Drenkhahn, G. 2002, A\& $A, 387,714$

Farris, B. D., Li, T. K., Liu, Y. T., \& Shapiro, S. L. 2008, Phys. Rev. D, 78, 024023

Kennel, C. F. \& Coroniti, F. V. 1984, ApJ, 283, 710

Lyubarsky, Y. E. 2005, MNRAS, 358, 113

Lyutikov, M. \& Uzdensky, D. 2003, ApJ, 589, 893

Masada, Y., Nagataki, S., Shibata, K., \& Terasawa, T. 2010, PAJS, 62, 1093

McKinney, J. C. \& Uzdensky, D. A. 2010, ArXiv e-prints

Shibata, M., Kiuchi, K., Sekiguchi, Y., \& Suwa, Y. 2011, Progress of Theoretical Physics, 125, 1255

Takahashi, H. R., Kudoh, T., Masada, Y., \& Matsumoto, J. 2011, ApJ, 739, L53

Watanabe, N. \& Yokoyama, T. 2006, ApJ, 647, L123

Zanotti, O. \& Dumbser, M. 2011, MNRAS, 418, 1004

Zenitani, S., Hesse, M., \& Klimas, A. 2010, ApJ, 716, L214

Zhang, B. \& Yan, H. 2011, ApJ 726, 90 